Res., Soc. Dev. 2019; 8(3):e983835

ISSN 2525-3409 | DOI: http://dx.doi.org/10.33448/rsd-v8i3.835

\title{
Depressão materna e fatores de risco associados
}

Maternal depression and associated risk factors

Depresión materna y factores de riesgo asociados

\section{Janaína Pereira Pretto Carlesso}

ORCID: https://orcid.org/0000-0001-8488-1906

Universidade Franciscana - UFN, Brasil.

E-mail: janapcarlesso@yahoo.com.br

Anaelena Bragança de Moraes

ORCID: https://orcid.org/0000-0002-6578-0613

Universidade Federal de Santa Maria (UFSM), Brasil.

Email: anaelena.bm@terra.com.br

Ana Paula Ramos de Souza

ORCID: https://orcid.org/0000-0003-4733-0632

Universidade Federal de Santa Maria (UFSM), Brasil.

Email: ana.souza@ufsm.br

Recebido: 10/12/2018 | Revisado: 12/12/2018 | Aceito: 18/12/2018 | Publicado: 21/12/2018

\section{Resumo}

Este estudo teve como objetivo analisar a frequência para a depressão pós-parto e os fatores de risco associados em uma amostra de mães de bebês nascidos em cidade de porte médio e arredores da região central do Rio Grande do Sul (RS). A pesquisa foi realizada em Hospital Escola no qual os bebês realizavam triagem auditiva neonatal, no período de março a maio de 2010. A amostra do estudo foi composta por 183 díades mães- bebês. Para a coleta de dados foi utilizado um roteiro de entrevista sobre informações socioeconômicas, demográficas, obstétricas e psicossociais e a aplicação do Inventário de Depressão de Beck (BDI). A maioria da amostra estudada não apresentou indícios de depressão pós-parto $(56,1 \%)$, sendo que os fatores de risco associados significativamente com a depressão foram à baixa renda familiar e o não planejamento da gravidez. As precárias condições socioeconômicas da mulher e o não planejamento da gestação foram os fatores que influenciaram significativamente no aparecimento da depressão no período pós-parto na amostra estudada.

Palavras-chave: depressão pós-parto; mães; fatores de risco. 


\section{Abstract}

This study aimed to analyze the frequency of postpartum depression and associated risk factors in a sample of mothers of infants born in a medium-sized city in the central region of Rio Grande do Sul (RS). The study was carried out in a School Hospital in which the infants underwent neonatal hearing screening, from March to May 2010. The study sample consisted of 183 infant-mother dyads. To collect data, an interview script was used on socioeconomic, demographic, obstetrical and psychosocial information and the Beck Depression Inventory (BDI). The majority of the sample studied did not present evidence of postpartum depression (56.1\%), and the risk factors associated with depression were low family income and no pregnancy planning. The precarious socioeconomic conditions of women and the non-planning of gestation were the factors that significantly influenced the onset of depression in the postpartum period in the sample studied.

Keywords: postpartum depression; mothers; risk factors.

\section{Resumen}

Este estudio tuvo como objetivo analizar la frecuencia para la depresión posparto y los factores de riesgo asociados en una muestra de madres de bebés nacidos en ciudad de tamaño medio y alrededores de la región central de Rio Grande do Sul (RS). La investigación se realizó en el Hospital Escuela en el que los bebés realizaban una clasificación auditiva neonatal en el período de marzo a mayo de 2010. La muestra del estudio fue compuesta por 183 díades madres lactantes. Para la recolección de datos se utilizó un guión de entrevista sobre informaciones socioeconómicas, demográficas, obstétricas y psicosociales y la aplicación del Inventario de Depresión de Beck (BDI). La mayoría de la muestra estudiada no presentó indicios de depresión posparto $(56,1 \%)$, siendo que los factores de riesgo asociados significativamente con la depresión fueron a la baja renta familiar y la no planificación del embarazo. Las precarias condiciones socioeconómicas de la mujer y la no planificación de la gestación fueron los factores que influenciaron significativamente en la aparición de la depresión en el período posparto en la muestra estudiada.

Palabras clave: depresión postparto; madres; factores de riesgo. 
Introdução

A depressão pós-parto (DPP), definida como transtorno do humor que se inicia, normalmente, nas primeiras quatro semanas após o parto, alcança sua intensidade máxima nos seis primeiros meses. Ela pode ser de intensidade leve e transitória ou agravar-se até uma neurose ou desordem psicótica (RODRIGUES ET AL, 2006; GALVÃO et al., 2015).

Cantilino et al (2010) e a American Psychiatric Association - APA (2013) caracterizam a DPP pode ser um conjunto de sintomas tais como: irritabilidade, perda da capacidade de sentir prazer, ansiedade, desânimo persistente, sentimento de culpa, entre outros. Tais sintomas têm início geralmente entre a quarta e sexta semana após o parto e interferem no bem-estar físico e mental da mulher, na saúde do lactente e na relação de ambos.

De acordo com as pesquisas realizadas, no Brasil, estudos apontam que a taxa de prevalência da DPP varia entre 12 e 37,1\% (FONSECA; SILVA; OTTA, 2010). Os sintomas mais comuns são: desânimo persistente, sentimentos de culpa, alterações do sono, idéias suicidas, temor de machucar o filho, redução do apetite e da libido, diminuição do nível de funcionamento mental e presença de ideias obsessivas.

Embora não se conheça claramente a etiologia da DPP, sabe-se que alguns fatores podem contribuir para a precipitação da depressão pós-parto, como: baixa condição socioeconômica; não aceitação da gravidez; maior número de gestações, de partos e de filhos vivos; menor tempo de relacionamento com o companheiro; história de problemas obstétricos; maior tempo para tocar no bebê após o nascimento; violência doméstica; pouco suporte por parte do companheiro; sobrecarga de tarefas; e experiência conflituosa da maternidade (BRITO et al., 2015). Há uma combinação de fatores biológicos, obstétricos, sociais e psicológicos que são apontados na determinação da depressão pós-parto.

Quanto aos fatores de risco é possível ainda que exista uma associação entre a ocorrência da depressão pós-parto e o pouco ou inexistente suporte oferecido por outras pessoas com quem a mãe mantém relacionamento; o não planejamento da gestação; o nascimento prematuro e a morte do neonato; a dificuldade em amamentar e as dificuldades no parto (BRITO et al., 2015).

Atualmente é possível detectar precocemente o surgimento da DPP através da aplicação de escalas sintomáticas de auto-relato, que identificam em diferentes níveis 
Res., Soc. Dev. 2019; 8(3):e983835

ISSN 2525-3409 | DOI: http://dx.doi.org/10.33448/rsd-v8i3.835

a gravidade da depressão. Neste estudo foi utilizado o Inventário de Depressão de Beck (BDI), que teve o objetivo de avaliar as manifestações comportamentais das mães estudadas no período pós-parto.

A partir de tais considerações, este estudo teve como objetivo analisar a frequência para a depressão pós-parto e os fatores de risco associados em uma amostra de mães de bebês nascidos em cidade de porte médio e arredores da região central do Rio Grande do Sul (RS).

\section{Metodologia}

Para a realização do cálculo amostral foi utilizada a prevalência da depressão materna no período pós-parto no Brasil, com base nos dados da Organização Mundial de Saúde (OMS, 2009), que é de 10\% a 15\%. A partir destas informações, este estudo assumiu a prevalência de depressão em $15 \%$ e a margem de erro de 5 pontos percentuais, estimando o tamanho da amostra mínima em 163 mães-bebês. Nesse estudo foram estudadas 183 mães-bebê, aproveitando a grande demanda de mães que buscam o serviço e já prevendo as possíveis perdas. Tal dimensão amostral se deu pela projeção da prevalência da depressão pós-parto na realidade de mães de bebês sem alterações biológicas no desenvolvimento, que buscam o serviço da triagem auditiva neonatal (TAN) no Hospital Universitário.

A pesquisa foi realizada em Hospital Universitário da região central do Rio Grande do Sul. A coleta de dados foi feita no período de março a maio de 2010 no ambulatório de Audiologia, local onde é realizada a triagem auditiva neonatal dos recém-nascidos da cidade e região. A amostra foi selecionada a partir dos seguintes critérios: todos os bebês pré-termo, a termo e pós-termo de ambos os sexos, com idade de 1 a 4 meses, aprovados na triagem auditiva neonatal e suas respectivas mães.

Foram considerados critérios de exclusão do estudo, os bebês que falhassem na triagem auditiva e que apresentassem malformações congênitas, síndromes genéticas ou infecção congênita detectadas no período neonatal, antes do início do estudo, pois estas por si só já representariam fatores de risco para o seu desenvolvimento. Esses critérios foram estabelecidos porque, além da depressão pós-parto, Estudaram-se os Indicadores Clínicos de Risco para o Desenvolvimento Infantil (IRDIs) nas díades (mães- bebês). Para este estudo, no entanto, serão abordados apenas os resultados relativos à depressão pós-parto. 
A coleta de dados foi realizada em dois momentos. O primeiro consistiu da entrevista com as mães e aplicação do Inventário de Depressão de Beck (BDI). No segundo foi feita uma filmagem da interação mãe-bebê para a análise dos Indicadores Clínicos de Risco para o Desenvolvimento Infantil (IRDIs). No primeiro momento as mães foram convidadas a participar do estudo pela psicóloga responsável da pesquisa, que explicou detalhadamente os propósitos do estudo. A seguir, as mães voluntariamente assinaram o Termo de Consentimento Livre e Esclarecido e o Termo para a criação de banco de imagens em duas vias. Esses documentos visavam informar as participantes, de forma sucinta, sobre os objetivos da pesquisa, sobre os dados dos pesquisadores responsáveis, além de assegurar o sigilo de suas identidades e de seus filhos.

A pesquisadora-responsável e seus colaboradores declararam-se comprometidos a zelar pelo cumprimento dos direitos dos sujeitos da pesquisa, garantindo a estes a privacidade e sigilo dos seus dados pessoais, o direito à informação continuada sobre o estudo, e o encaminhamento para auxílio de saúde quando fosse necessário. As mães foram entrevistadas numa única sessão, em uma sala especialmente preparada para tal finalidade, com duração de aproximadamente 30 minutos.

Para realização a da entrevista, foi utilizado um roteiro com o objetivo de investigar as informações socioeconômicas (renda familiar, escolaridade, ocupação), demográficas (idade, estado civil, número de filhos), obstétricas (número de gestações, número de partos, história de aborto, partos prematuros, tipo de parto, número de consultas pré-natais, planejamento da gestação, peso do recém-nascido, intercorrências com o recém-nascido, idade gestacional, tipo de aleitamento) e psicossociais (suporte social, histórico de depressão materna e experiência da maternidade).

No roteiro também foram incluídas duas questões abertas, que investigaram a crise situacional e os antecedentes psiquiátricos. Este roteiro foi baseado na Entrevista sobre a Experiência da Maternidade (Grupo de Pesquisa em Infância, Desenvolvimento e Psicopatologia, \& Núcleo de Infância e Família, 2003), instrumento utilizado no projeto de pesquisa do Instituto de Psicologia da UFRGS.

$\mathrm{Na}$ entrevista foi solicitado que as mães falassem sobre diversos aspectos relacionados ao desenvolvimento do bebê e à experiência da maternidade. O objetivo 
ISSN 2525-3409 | DOI: http://dx.doi.org/10.33448/rsd-v8i3.835

da entrevista foi investigar a evolução dos afetos e vivências emocionais com relação à maternidade, habilidades e características emocionais do bebê, os sentimentos e atitudes no desempenho do papel materno, a forma como esses padrões afetam a relação mãe-filho e o desenvolvimento da criança. Examinou-se também a ocorrência de eventos estressantes.

Realizada a entrevista, as mães foram convidadas a responder o BDI com a finalidade de investigar o risco de depressão materna no período pós-parto. Por se tratar de participantes que conduziam seus bebês no colo, a pesquisadora anotou as respostas da entrevista e também da escala de avaliação da depressão. As respostas do BDI foram corrigidas pela psicóloga responsável pelo estudo.

O BDI é uma escala sintomática de auto-relato, composta por 21 itens com diferentes alternativas de resposta a respeito de como o sujeito tem se sentido recentemente, e que correspondem a diferentes níveis de gravidade da depressão. A soma dos escores dos itens individuais fornece um escore total, que por sua vez constitui um escore dimensional da intensidade da depressão, que pode ser classificado nos seguintes níveis: em mínimo (0 a 11 pontos), leve (12 a 19), moderado (20 a 35) e grave (36 a 63). A versão em português do Inventário Beck de Depressão resultou de uma formulação consensual da tradução do original em inglês, com a colaboração de quatro psicólogos clínicos, quatro psiquiatras e uma tradutora, sendo testada junto com a versão em inglês em 32 pessoas bilíngües, com três dias de intervalo e variando a ordem da apresentação dos dois idiomas nas duas metades da amostra (BECK, 2001).

As mães que apresentaram depressão, pela avaliação do BDI, foram convidadas a participar de grupos terapêuticos e também de acompanhamento psicológico individual. Este estudo foi aprovado pelo Comitê de Ética em Pesquisa da Universidade e pelo departamento de ensino e pesquisa do Hospital (HU), em seus aspectos éticos e metodológicos de acordo com as Diretrizes estabelecidas na Resolução 196/96 e complementares do Conselho Nacional de Saúde, sob o número do CAEE n. 0284.0.243.000-09.

Para avaliar a relação entre a depressão pós-parto e seus fatores de risco associados, foram utilizados os testes não-paramétricos U de Mann-Whitney e KruskalWallis, seguido de comparações múltiplas (teste post-hoc). Admitiu-se nível de significância estatística de 0,05 . As respostas obtidas no roteiro de entrevista foram 
categorizadas em variáveis que foram agrupadas em sub-variavéis qualitativas: socioeconômicas (renda familiar, escolaridade, ocupação), demográficas (idade, estado civil, número de filhos), obstétricas (número de gestações, número de partos, história de aborto, partos prematuros, tipo de parto, número de consultas pré-natais, planejamento da gestação, peso do recém-nascido, intercorrências com o recémnascido, tipo de aleitamento) e psicossociais (suporte social, histórico de depressão materna e experiência da maternidade).

Todas as variáveis foram codificadas sendo construído o banco de dados em uma planilha do Excel. Os valores do BDI foram analisados em função das categorias das variáveis. A análise estatística foi realizada utilizando-se o aplicativo computacional STATISTICA 9.0.

\section{Resultados e discussões}

A maioria $(56,1 \%)$ das mães avaliadas pelo BDI não apresentou indícios de depressão pós-parto (DPP). Na Tabela 1 pode-se observar as frequências e seus percentuais para cada faixa de valor das variáveis (sub-variáveis socioeconômicas e demográficas), bem como suas frequências por faixa de valores do BDI. Também são apresentados os resultados dos testes estatísticos.

O nível de escolaridade mais frequente na amostra estudada foi o ensino médio, tendo predomínio em 91 mulheres (49,7\%). Cem mulheres $(54,6 \%)$ eram donas de casa, sendo que as outras oitenta e três $(45,4 \%)$ exerciam ocupações fora do lar. A maioria $(63,4 \%)$ das mães tinha idade entre 20 e 34 anos. Cento e cinquenta e seis $(85,2 \%)$, era casada ou vivia em união consensual, na qual o pai e a mãe habitavam o mesmo lar desde o momento do parto, independendo do estado civil ou religioso. 
Res., Soc. Dev. 2019; 8(3):e983835

ISSN 2525-3409 | DOI: http://dx.doi.org/10.33448/rsd-v8i3.835

Tabela 1- Frequência das variáveis socioeconômicas e demográficas e da depressão pós-parto (BDI), em uma amostra ( $\mathrm{n}=183)$ de mães de bebês nascidos em cidade de porte médio e arredores da região central do Rio Grande do Sul (RS), 2010.

\begin{tabular}{|c|c|c|c|c|c|c|}
\hline \multirow{2}{*}{$\begin{array}{l}\text { Variáveis } \\
\text { Socioeconômicas }\end{array}$} & \multirow[b]{2}{*}{ Total (\%) } & \multicolumn{5}{|c|}{$B D I(\%)$} \\
\hline & & Minimo & Leve & Moderado & Grave & Valor de $p^{*}$ \\
\hline $\begin{array}{l}\text { Renda familiar (s.m.) } \\
\text { menos de } 1 \\
1 \text { a } 2,9 \\
3 \text { ou mais }\end{array}$ & $\begin{array}{r}53(29,9) \\
103(58,2) \\
21(11,9)\end{array}$ & $\begin{array}{l}27(50,9) \\
75(72,8) \\
15(71,4)\end{array}$ & $\begin{array}{r}10(18,9) \\
18(17,5) \\
5(23,8)\end{array}$ & $\begin{array}{r}13(24,5) \\
7(6,8) \\
1(4,8)\end{array}$ & $\begin{array}{l}3(5,7) \\
3(2,9) \\
0(0,0)\end{array}$ & 0,021 \\
\hline $\begin{array}{l}\text { Escolaridade } \\
\text { Fundamental ou analfabeto } \\
\text { Médio } \\
\text { Superior }\end{array}$ & $\begin{array}{r}78(42,9) \\
91(50,0) \\
13(7,1)\end{array}$ & $\begin{array}{r}47(60,2) \\
60(66,7) \\
11(84,2)\end{array}$ & $\begin{array}{r}17(21,9) \\
18(20,0) \\
0(0,0)\end{array}$ & $\begin{array}{r}8(10,6) \\
11(12,2) \\
2(15,3)\end{array}$ & $\begin{array}{l}6(7,6) \\
1(1,1) \\
0(0,0)\end{array}$ & 0,214 \\
\hline $\begin{array}{l}\text { Profissão } \\
\text { Dona de casa } \\
\text { Outra }\end{array}$ & $\begin{array}{r}100(54,6) \\
83(45,4) \\
\end{array}$ & $\begin{array}{l}54(54,0) \\
65(79,2) \\
\end{array}$ & $\begin{array}{l}24(24,0) \\
11(13,4) \\
\end{array}$ & $\begin{array}{r}16(16,0) \\
5(6,10) \\
\end{array}$ & $\begin{array}{l}6(6,0) \\
1(1,2) \\
\end{array}$ & $<0,01$ \\
\hline Demográficas & & & & & & \\
\hline $\begin{array}{l}\text { Idade } \\
\text { Menor que } 20 \text { anos } \\
20 \text { a } 34 \text { anos } \\
35 \text { em diante }\end{array}$ & $\begin{array}{r}34(18,6) \\
116(63,4) \\
33(18,0)\end{array}$ & $\begin{array}{l}20(58,8) \\
77(67,0) \\
22(66,6)\end{array}$ & $\begin{array}{r}8(23,5) \\
19(16,5) \\
8(24,2)\end{array}$ & $\begin{array}{r}5(14,7) \\
14(12,1) \\
2(6,0)\end{array}$ & $\begin{array}{l}1(2,9) \\
5(4,3) \\
1(3,0)\end{array}$ & 0,672 \\
\hline $\begin{array}{l}\text { Estado civil } \\
\text { Casada ou união consensual } \\
\text { Solteira }\end{array}$ & $\begin{array}{r}156(85,2) \\
27(14,8)\end{array}$ & $\begin{array}{r}106(68,3) \\
13(48,1)\end{array}$ & $\begin{array}{r}31(20 \mid 0) \\
4(14,8)\end{array}$ & $\begin{array}{r}11(7,1) \\
10(37,0)\end{array}$ & $\begin{array}{l}7(4,5) \\
0(0,0)\end{array}$ & 0,933 \\
\hline $\begin{array}{l}\mathbf{N}^{\circ} \text { de filhos } \\
1 \\
2 \\
3 \text { ou mais }\end{array}$ & $\begin{array}{l}87(47,5) \\
54(29,5) \\
42(23,0)\end{array}$ & $\begin{array}{l}58(66,6) \\
39(72,2) \\
22(53,6)\end{array}$ & $\begin{array}{r}17(19,5) \\
9(16,6) \\
9(21,9)\end{array}$ & $\begin{array}{r}10(11,4) \\
5(9,2) \\
6(14,6)\end{array}$ & $\begin{array}{l}2(2,3) \\
1(1,8) \\
4(9,7)\end{array}$ & 0,160 \\
\hline Total & $183(100,0)$ & $119(65,0)$ & $35(19,1)$ & $21(10,5)$ & $7(3,8)$ & \\
\hline
\end{tabular}

Fonte: Banco de dados dos autores.

Quanto à avaliação da depressão pelo BDI, pode-se observar na Tabela 1, a frequência dos níveis de indícios de depressão pós-parto na amostra estudada. Quando se avaliou os níveis do BDI em função da sub- variável socioeconômica (renda familiar, escolaridade e profissão), foi encontrada diferença significativa nos níveis do BDI entre as faixas de 1 a 2,9 salários mínimos e 3 ou mais salários mínimos. Sendo que, quanto menor a renda familiar, maior a frequência de depressão pós-parto.

Em relação aos níveis de depressão do BDI e a sub- variável demográfica (idade materna, estado civil e número de filhos), não foram aferidos valores estatisticamente significativos. Nesta tabela pode-se verificar também que o nível de depressão é significativamente maior entre as donas de casa do que em relação às mulheres que trabalham fora.

$\mathrm{Na}$ Tabela 2 pode-se observar as frequências e seus percentuais para cada faixa de valor das variáveis (sub-variáveis obstétricas e psicossociais), bem como suas frequências por faixa de valores do BDI. Também são apresentados os resultados dos testes estatísticos. Noventa e cinco mulheres $(44,8 \%)$ não planejaram a gestação. A maioria das mães estudadas $(72,1 \%)$ não apresentou histórico de depressão. Cento e 
Res., Soc. Dev. 2019; 8(3):e983835

ISSN 2525-3409 | DOI: http://dx.doi.org/10.33448/rsd-v8i3.835

dezoito mulheres $(55,6 \%)$, não estavam acometidas de alterações emocionais maternas.

Tabela 2 - Frequência das variáveis obstétricas e psicossociais e da depressào pós-parto (BDI), em uma amostra ( $n=183$ ) de màes de bebês nascidos em cidade de porte médio e arredores da regiâo central do Rio Grande do Sul (RS), 2010.

\begin{tabular}{|c|c|c|c|c|c|c|}
\hline Variaveis & & & $B D I(\%)$ & & & \\
\hline Obstetricas & Total (\%) & Dinima & Leve & Moderado & Grave & Valor dep \\
\hline $\begin{array}{l}N^{2} \text { de gestacóes } \\
1 \\
2 \text { a } 4 \\
5 \text { ou mais }\end{array}$ & $\begin{array}{r}76(41,5) \\
90(49,2) \\
17(9,3) \\
\end{array}$ & $\begin{array}{r}51(67,1) \\
59(66,2) \\
9(52,4) \\
\end{array}$ & $\begin{array}{l}14(18,4) \\
17(19,1) \\
4(23,5)\end{array}$ & $\begin{array}{r}10(13,1) \\
8(8,9) \\
3(17,6) \\
\end{array}$ & $\begin{array}{l}1(1,3) \\
5(5,6) \\
1(5,8)\end{array}$ & 0,523 \\
\hline $\begin{array}{l}N^{9} \text { de partos } \\
1 \\
2 \text { a } 4 \\
5 \text { ou mais }\end{array}$ & $\begin{array}{r}85(46,4) \\
84(45,9) \\
14(7,7)\end{array}$ & $\begin{array}{r}57(67,0) \\
53(63,8) \\
9(64,2)\end{array}$ & $\begin{array}{l}17(20,0) \\
16(19,2) \\
2(14,2)\end{array}$ & $\begin{array}{r}10(11,7) \\
9(10,8) \\
2(14,2)\end{array}$ & $\begin{array}{l}10(11,7) \\
9(10,8) \\
2(14,2)\end{array}$ & 0,906 \\
\hline $\begin{array}{l}\text { Historia de aborto } \\
\text { Sim } \\
\text { Nao }\end{array}$ & $\begin{array}{r}33(18,0) \\
150(82,0)\end{array}$ & $\begin{array}{r}19(57,5) \\
100(67,1)\end{array}$ & $\begin{array}{r}19(57,5) \\
100(67,1)\end{array}$ & $\begin{array}{r}4(12,1) \\
17(11,4)\end{array}$ & $\begin{array}{l}3(9,0) \\
4(2,6)\end{array}$ & 0,313 \\
\hline $\begin{array}{l}\text { Partos prematuros } \\
\text { Sim } \\
\text { Näo } \\
\end{array}$ & $\begin{array}{r}51(27,9) \\
132(72,1) \\
\end{array}$ & $\begin{array}{l}29(58,0) \\
90(68,1)\end{array}$ & $\begin{array}{r}7(14,0) \\
28(21,2) \\
\end{array}$ & $\begin{array}{r}10(20,0) \\
11(8,3) \\
\end{array}$ & $\begin{array}{r}4(8,0) \\
3(2,27) \\
\end{array}$ & 0,122 \\
\hline $\begin{array}{l}\text { Tipo de parto } \\
\text { Vaginal } \\
\text { Cesarea }\end{array}$ & $\begin{array}{r}68(37,2) \\
115(62,8)\end{array}$ & $\begin{array}{l}44(64.7) \\
75(65.7)\end{array}$ & $\begin{array}{l}14(20,5) \\
21(18,4)\end{array}$ & $\begin{array}{r}10(14,7) \\
11(9,6)\end{array}$ & $\begin{array}{l}0(0,0) \\
7(6,1)\end{array}$ & 0,968 \\
\hline $\begin{array}{l}N^{\circ} \text { cousultas pre-uatais } \\
\text { Nenhum } \\
\text { Ate } 5 \\
6 \text { ou mais }\end{array}$ & $\begin{array}{r}0(0,0) \\
21(11,5) \\
162(88,5)\end{array}$ & $\begin{array}{r}0(0,0) \\
15(71,4) \\
104(54,6)\end{array}$ & $\begin{array}{c}0(0,0) \\
2(9,5) \\
33(20,5)\end{array}$ & $\begin{array}{r}0(0,0) \\
2(9,5) \\
19(11,8)\end{array}$ & $\begin{array}{l}0(0,0) \\
2(9,5) \\
5(3,1)\end{array}$ & 0,793 \\
\hline $\begin{array}{l}\text { Gestaçáo } \\
\text { Planejada } \\
\text { Náo planejada } \\
\text { Indesejada }\end{array}$ & $\begin{array}{r}81(44,3) \\
95(51,9) \\
7(3,8) \\
\end{array}$ & $\begin{array}{r}62(76,5) \\
54(57,4) \\
3(42,8) \\
\end{array}$ & $\begin{array}{r}12(14,8) \\
20(21,2) \\
3(42,8) \\
\end{array}$ & $\begin{array}{r}4(4,9) \\
16(17,0) \\
1(14,2) \\
\end{array}$ & $\begin{array}{l}3(3,7) \\
4(4,2) \\
0(0,0)\end{array}$ & 0,015 \\
\hline $\begin{array}{l}\text { Baixo peso } \\
\text { Sim } \\
\text { Nao }\end{array}$ & $\begin{array}{r}15(8,2) \\
168(91,8) \\
\end{array}$ & $\begin{array}{r}12(8,0) \\
10764,0)\end{array}$ & $\begin{array}{r}1(6,6) \\
34(20,3) \\
\end{array}$ & $\begin{array}{r}1(6,6) \\
20(11,9) \\
\end{array}$ & $\begin{array}{l}1(6,6) \\
6(3,5) \\
\end{array}$ & 0,380 \\
\hline $\begin{array}{l}\text { Intercorrëncias com } \mathrm{RV} \\
\text { Sim } \\
\mathrm{Na \textrm {o }}\end{array}$ & $\begin{array}{r}60(33,3) \\
120(66,7)\end{array}$ & $\begin{array}{l}38(63,3) \\
81(67,5)\end{array}$ & $\begin{array}{l}13(21,6) \\
21(17,5)\end{array}$ & $\begin{array}{r}7(11,6) \\
13(10,8)\end{array}$ & $\begin{array}{l}2(3,3) \\
5(4,1)\end{array}$ & 0,709 \\
\hline $\begin{array}{l}\text { Tipo de aleitamento } \\
\text { Matemo Exchsivo } \\
\text { Predominante Compl } \\
\text { Artificial } \\
\text { + de l tipo de aleitanento }\end{array}$ & $\begin{array}{r}127(69,8) \\
0(0,0) \\
18(9,9) \\
37(20,3) \\
\end{array}$ & $\begin{array}{r}86(67,7) \\
0(0,0) \\
13(72,2) \\
20(54,0) \\
\end{array}$ & $\begin{array}{r}24(18,9) \\
0(0,0) \\
4(22,2) \\
7(18,9) \\
\end{array}$ & $\begin{array}{r}12(9,4) \\
0(0,0) \\
1(5,5) \\
8(21,6) \\
\end{array}$ & $\begin{array}{l}5(3,9) \\
0(0,0) \\
0(0,0) \\
2(5,4)\end{array}$ & 0,250 \\
\hline Psicossociais & & & & & & \\
\hline $\begin{array}{l}\text { Suporte social } \\
\text { Nenhum } \\
\text { Marido } \\
\text { Mage } \\
\text { Outros } \\
+ \text { de } 1 \text { cuidador }\end{array}$ & $\begin{array}{r}34(18,7) \\
44(24,2) \\
42(23,1) \\
17(9,3) \\
45(24,7)\end{array}$ & $\begin{array}{r}17(50,0) \\
29(65,9) \\
31(73,8) \\
9(52,9) \\
33(73,3)\end{array}$ & $\begin{array}{l}13(3,8,2) \\
6(13,6) \\
6(14,2) \\
4(23,5) \\
6(13,3)\end{array}$ & $\begin{array}{l}4(11,7) \\
6(13,6) \\
5(11,9) \\
2(11,7) \\
4(8,8)\end{array}$ & $\begin{array}{r}0(0,0) \\
3(6,8) \\
0(0,0) \\
2(11,7) \\
2(4,4)\end{array}$ & 0,121 \\
\hline $\begin{array}{l}\text { Depressáo ma terna } \\
\text { Simn } \\
\text { Não }\end{array}$ & $\begin{array}{r}30(16,4) \\
153(83,0)\end{array}$ & $\begin{array}{c}16(53,3) \\
103(67,7)\end{array}$ & $\begin{array}{r}7(23,3) \\
28(18,4)\end{array}$ & $\begin{array}{r}4(13,3) \\
17(11,1)\end{array}$ & $\begin{array}{r}3(10,0) \\
4(2,6)\end{array}$ & 0,159 \\
\hline $\begin{array}{l}\text { Experiéncia matema } \\
\text { Nenh. alt, emoc. } \\
\text { Alt. emoc. leve } \\
\text { Alt. emoc. moderada } \\
\text { Alt. emoc. grave } \\
\text { Total }\end{array}$ & $\begin{array}{r}118(64,4) \\
34(18,6) \\
21(11,5) \\
10(5,5) \\
183(100,0)\end{array}$ & $\begin{array}{r}93(79,4) \\
18(52,9) \\
8(38,1) \\
0(0,0) \\
119(65,0)\end{array}$ & $\begin{array}{r}21(17,9) \\
11(32,3) \\
2(9,5) \\
1(10,0) \\
35(19,1)\end{array}$ & $\begin{array}{r}3(2,5) \\
5(14,7) \\
11(52,3) \\
2(20,0) \\
21(10,5)\end{array}$ & $\begin{array}{r}0(0,0) \\
0(0,0) \\
0(0,0) \\
7(70,0) \\
7(3,8)\end{array}$ & $<0,01$ \\
\hline
\end{tabular}

-Testes: Ude Mann-Whitney e Krukkal-Wallis; BDI-Inventário de Depressâo de Beck RN = recém-nascido; Alt emoc. = alteraçâo emocional Exchidos os casos sem informaçäo: intercoméncias com RN (3); tipo de aleitamento (1); suporte social (1).

Fonte: Banco de dados dos autores.

Ainda nesta tabela, pode-se observar que, quando se avaliaram os níveis do BDI em função da sub-variável obstétrica, encontrou-se diferença estatisticamente significativa nos níveis do BDI quanto ao planejamento da gestação $(\mathrm{p}=0,015)$, sendo que nas comparações múltiplas entre gestação planejada e não-planejada foi obtido o 
p-valor de 0,065, entretanto, não significativo ao nível de significância de $5 \%$.

Ao se avaliar a sub-variável psicossocial, encontrou-se diferença significativa nos níveis do BDI entre os graus de alteração emocional materna. Os níveis do BDI diferem entre as mães sem alteração emocional e mães com alteração emocional moderada ou grave e entre mães com alteração emocional leve e grave.

A depressão pós-parto avaliada pelo BDI não esteve presente na maioria das mulheres desta pesquisa, resultado semelhante ao estudo realizado por (FRIZZO; PICCININI, 2007). Neste estudo pode-se observar que a variável socioeconômica que apresentou associação com a depressão pós-parto foi a baixa renda familiar, resultado semelhante encontrado em outros estudos realizados (RICH-EDWARDS ET AL, 2006; MOARES ET AL, 2006; RUSCHI ET AL,2007; SEGRE ET AL, 2007; TANNOUS ET AL, 2008; ALIANE; MAMEDE; FURTADO, 2011; KERBER; FALCETO; FERNANDES, 2011; SILVA ET AL, 2012; ATIF ET AL, 2015).

Há uma pesquisa brasileira (Moraes et al, 2006), que relata um predomínio entre 32 e $38 \%$ de depressão puerperal em mulheres de baixa renda, o mesmo é verificado em um estudo internacional de (Lau; Wong; Chan, 2010). Este aspecto é também ressaltado nos estudos de (Zanotti et al, 2003) que afirmam que a baixa educação, a pobreza e uma relação familiar conflituosa concorrem fortemente para o aparecimento da depressão pósparto e muitos casos estão associados ao fato de a gravidez não ter sido planejada ou desejada (Beck, 2002; Saraiva; Coutinho, 2007). Neste estudo a gravidez não planejada também surge como fator obstétrico estatisticamente significante para o surgimento da depressão pós-parto, o que confirma vários dos estudos revisados.

As demais variáveis demográficas e obstétricas investigadas nesse estudo, não apresentaram correlação estatisticamente significante com a presença de depressão pósparto, resultado semelhante ao estudo realizado por (Mosso et al, 2008). Há estudos que encontraram a correlação entre algumas variáveis investigadas neste estudo realizado, como idade (Schwengber; Piccinini, 2003; Monteiro, 2010), estado civil (Frizzo; Piccinini, 2007; Monteiro, 2010), com a depressão pós-parto o que difere dos resultados desta pesquisa.

Talvez um dos fatores psicossociais que conspiraram para a ausência de alterações emocionais e de estado depressivo na amostra estudada é o fato de a maior parte das mães possuírem uma rede de apoio social significativa. Possivelmente, houve tanto uma minimização do processo depressivo nas mães, quanto à presença de proteção a elas e aos 
bebês quando este apresentou-se como moderado ou grave. É possível propor tal hipótese em função de vários estudos realizados tanto no Brasil quanto em outros países, que afirmam que a rede de apoio pode impedir ou minimizar os efeitos da depressão pós-parto no exercício da função materna (CHANDRAN ET AL, 2002; CRUZ; SIMÕES; FAISAL-CURY, 2005; MATTAR ET AL, 2007; ELGAR ET AL, 2007; MAMUN ET AL, 2009; MONTEIRO, 2010; KONRADT ET AL, 2011; MELLO JR ET AL, 2012; SILVA ET AL, 2012; TOPIWALA ET AL, 2012).

Acerca disso, cabe ressaltar a importância da rede de apoio ás mães durante o puerpério, pois é este é um período de grandes transformações e novas adaptações na vida da mulher, pois a mesma assume um novo papel e tem de dar conta de um universo de demandas do bebê, por isso é necessário que as mães estejam amparadas socialmente pelo cônjuge, familiares para que os sintomas depressivos sejam minimizados nessa fase.

\section{Considerações finais}

O estudo realizado apontou que a Depressão pós-parto (DPP) se constitui uma problemática de Saúde Pública, que pode ter repercussões negativas e interferir diretamente na relação da díade mãe com o bebê. A partir da pesquisa realizada podese identificar, apesar da etiologia da DPP não ser definida claramente, aponta-se que multifatoriedade, como principal fator de risco para seu desenvolvimento.

A análise do estudo apontou que a maior parte das mães apresentou depressão mínima, não houve alteração na experiência da maternidade, o que se apresentou como estatisticamente significante, ou seja, a ausência de dificuldades para exercer a função materna foi relevante na amostra porque havia poucos casos de depressão pós-parto e, possivelmente, porque havia suporte social e desejo de ocupar tal posição.

Este estudo não pode confirmar em larga escala, portanto, o resultado de vários estudos que demonstram que mães deprimidas podem sentir-se incapazes de cuidar do bebê e exercer seu papel (ZAGONEL ET AL, 2003; SCHWENGBER; PICCININI, 2003, MONTEIRO, 2010). As mães podem sentir frequentemente menos ligadas emocionalmente a seus bebês, mais dependentes e isoladas socialmente, e possuem menor confiança e satisfação com o desempenho da função materna (SCHWENGBER; PICCININI, 2003).

Sugerem-se mais estudos e reflexões sobre a temática estudada, pois é sempre 
Res., Soc. Dev. 2019; 8(3):e983835

ISSN 2525-3409 | DOI: http://dx.doi.org/10.33448/rsd-v8i3.835

relevante reforçar a necessidade de investigar a presença dos fatores de risco associados á DPP, desde as consultas de pré-natal, oportunizando uma atenção integral à saúde da mulher, com ações de prevenção e promoção, visando minimizar o aparecimento da depressão materna e seus possíveis efeitos deletérios sobre a relação da díade mãe-bebê no período pós-parto.

\section{Referências}

ALIANE, P.P; MAMEDE, M.V; FURTADO, E.F. Revisão Sistemática sobre fatores de risco associados à depressão pós-parto. Psicol Pesq.v.n2, 146-55, 2011. Disponível em: http://pepsic.bvsalud.org/scielo.php?script=sci_arttext\&pid=S1982-12472011000200007. Acesso: 18/12/2018.

AMERICAN PSYCHIATRIC ASSOCIATION. Diagnostic and Statistical Manual of Mental Disorders. Washington, DC: American Psychiatric Association; 2013. Disponível em: http://dsm.psychiatryonline.org/doi/book/10.1176/appi.books.9780890425596 16/12/2018.

ATIF N, LOVELL K, RAHMAN A. Maternal mental health: the missing "m" in the global maternal and child health agenda. Semin Perinatol. 2015. Disponível em: https://www.ncbi.nlm.nih.gov/pubmed/26164538 . 16/12/2018.

BECK, C.T. A depressão pós-parto: a meta-síntese. Qual Health Res. PubMed. Qual Health Res, v.2, 453-47, 2002. Disponível em: https://journals.sagepub.com/doi/abs/10.1177/104973202129120016 Acesso: 16/12/2018.

BECK, C. Predictors of postpartum depression: an update. Nurs Res, v.50, n.5, 275-85, 2001. Disponível em: https://www.ncbi.nlm.nih.gov/pubmed/11570712. Acesso: $16 / 12 / 2018$.

BRITO, C.N. O ET AL . Depressão pós-parto entre mulheres com gravidez não pretendida. Rev. Saúde Pública, São Paulo, v. 49, n.33, 2015. Disponível em: http://www.scielo.br/pdf/rsp/v49/pt_0034-8910-rsp-S0034-89102015049005257.pdf Acesso: $16 / 12 / 2018$.

CANTILINO, A; ZAMBALDI, C.F; SOUGEY, E.B; RENNÓ, J.R. Postpartum psychiatric disorders. Rev Psiquiatr Clín. v.37, n.6, 288-94,2010. Disponível em: http://www.scielo.br/scielo.php?script=sci_arttext\&pid=S0101-60832010000600006 Acesso: $16 / 12 / 2018$.

COSTA, R; PACHECO, A; FIGUEIREDO, B. Prevalência e preditores de sintomatologia depressiva após o parto. Rev Psiquiatra Clín, v.34, n.4, 157-65, 2007. Disponível em: http://www.scielo.br/pdf/rpc/v34n4/a01v34n4.pdf Acesso: 16/12/2018.

CRUZ, E.B.S; SIMÕES, G.L; FAISAL-CURY, A. Rastreamento da depressão pós-parto em mulheres atendidas pelo Programa de Saúde da Família. Rev Bras Ginecol Obstetr, v.27, n.4, 181-8, 2005. Disponível em: http://www.scielo.br/scielo.php?script=sci_arttext\&pid=S010072032005000400004\&lng=en\&nrm=iso\&tlng=pt. Acesso: 16/12/2018. 
EBERHARD-GRAN, M; ESKILD, A; TAMBS, K; SAMUELSEN, S.O; OPJORDSMOEN, S. Depression in postpartum and non-postpartum women: prevalence and risk factors. Acta Psychatr Scand, v.106, n.6, 426-33, 2002. Disponível em: https://www.ncbi.nlm.nih.gov/pubmed/12392485. Acesso: 16/12/2018.

ELGAR, F.J ET AL. Maternal and paternal depressive symptoms and child maladjustment: The mediating role of parental behavior. Journal of Abnormal Child Psychology, v. 35, 943-955, 2007. Disponível em: https://www.ncbi.nlm.nih.gov/pubmed/17577659 Acesso: 16/12/2018.

FONSECA, V.R.J.R.M.; SILVA, G.A.; OTTA, E. Relação entre depressão pós-parto e disponibilidade emocional materna. Cad. Saúde Pública, v.26, n.4, p.738-746, 2010. Disponível em: $\quad$ http://www.scielo.br/scielo.php?pid=S0102311X2010000400016\&script=sci_abstract\&tlng=pt Acesso: 16/12/2018.

FRIZZO, G.B; PICCININI, C.A. Depressão maternal e a interação triádica pai-mãebebê. Psicologia: Reflexão e Crítica, v.20, n.3, 351-360, 2007. Disponível em: http://www.scielo.br/scielo.php?script=sci_arttext\&pid=S0102-79722007000300002 Acesso: 16/12/2018.

GALVÃO, A.C.C et al. Prevalência de depressão pós-parto e fatores associados: uma revisão integrativa. ReOnFacema, v1.n1,54-58,2015. Disponível em: http://www.facema.edu.br/ojs/index.php/ReOnFacema/article/view/3/ Acesso: 16/12/2018.

Grupo de Pesquisa em Infância, Desenvolvimento e Psicopatologia, \& Núcleo de Infância e Família. Entrevista sobre experiência da maternidade. Manuscrito não-publicado, Universidade Federal do Rio Grande do Sul, Instituto de Psicologia, Porto Alegre, RS. (2003g). Disponível em: http://www.ufrgs.br/psicologia/nucleos-elaboratorios/nudif/apresentacao Acesso: 16/12/2018.

GUILLAND, R; MONTEIRO, J.K. Jovens e desemprego: estado da arte, Rev Psicol, Organ Trab. 2010. Disponível em: https://periodicos.ufsc.br/index.php/rpot/article/view/22215 Acesso: 16/12/2018.

KERBER, S.R.; FALCETO, O.G.; FERNANDES, C.L.C. Problemas conjugais e outros fatores associados a transtornos psiquiátricos do pós-parto. Rev Bras Ginecol Obstet, v.33, n.6, p.281-287, 2011. Disponível em: http://www.scielo.br/scielo.php?pid=S010072032011000600004\&script=sci_abstract\&tlng=es Acesso: 16/12/2018.

KONRADT, C.E. et al. Depressão pós-parto e percepção de suporte social durante a gestação. Rev. Psiquiatr. Rio Grande do Sul, v.33, n.2, p.76-79, 2011. Disponível em: http://www.scielo.br/scielo.php?pid=S0101$\underline{81082011000200003 \& \text { script=sci_abstract\&tlng=pt Acesso: 16/12/2018. }}$

LANCASTER, C.A ET AL.Risk factors for depressive symptoms during pregnancy, Am J Obstet Gynecol. V.22, n.1, 5-14.2010. Disponível em: https://www.ncbi.nlm.nih.gov/pmc/articles/PMC2919747/ Acesso: 16/12/2018.

LAU, Y; WONG, D.F; CHAN, K.S. The utility of screening for perinatal depression in the second trimester among Chinese: a three-wave prospective longitudinal study. Arch 
Womens Ment Health, v.13, n.2, 153-64, 2010. Disponível em: https://www.ncbi.nlm.nih.gov/pmc/articles/PMC2953626/ Acesso: 16/12/2018.

MATTAR, R ET AL. A violência doméstica como indicador de risco no rastreamento da depressão pós-parto. Rev Bras Ginecol Obstetr, v.29, n.9, 470-7, 2007. Disponível em: http://www.scielo.br/scielo.php?pid=S0100-

72032007000900006\&script=sci_abstract\&tlng=pt Acesso: 16/12/2018.

MAMUN, A. A; CLAVARINO, A. M; NAJMAN, J. M; WILlIAMS, G. M; O'CALLAGHAN, M. J; BOR, W. Maternal depression and the quality of marital relationship: A 14-year prospective study. Journal of Women's Health, v.18, n.12, 2023-31, 2009. Disponível em: https://www.ncbi.nlm.nih.gov/pubmed/20044866 Acesso: 16/12/2018.

MELO, EF J.R ET AL The prevalence of perinatal depression and its associated factors in two different settings in Brazil. Journal of Affective Disorders, v.136, n.3, 1204-8, 2012. Disponível em: https://www.ncbi.nlm.nih.gov/pubmed/22169251 Acesso: 16/12/2018.

MONTEIRO, I.S. O contributo das experiências familiares, vinculação e apoio social para a depressão no adulto, [tese]. Braga, Portugal: Universidade de Minho; 2010. Disponível em: http://repositorium.sdum.uminho.pt/handle/1822/10872 Acesso: 16/12/2018.

MORAES, I.G.S; PINHEIRO, R.T; SILVA, R.A; HORTA, B.L; SOUSA, P.L.R; FARIA, A.D. Prevalência da depressão pós-parto e fatores associados. Rev Saúde Publica, v.40, 65-70, 2006.

Disponível em: http://www.scielo.br/scielo.php?script=sci_arttext\&pid=S0034-89102006000100011 Acesso: 16/12/2018.

MOSSO, F.T. ET AL. Prevalência de depressão pós-parto em puérperas de Maringá. Revista Saúde e Pesquisa, v.1, n.3, 251-257, 2008. Disponível em: http://periodicos.unicesumar.edu.br/index.php/saudpesq/article/view/902. $\quad$ Acesso: $16 / 12 / 2018$.

RICH-EDWARDS, J.W; KLEINMAN, K; ABRAMS, A; HARLOW, B.L; MCLAUGHLIN, T.J; JOFFE, H; GILLMAN, M.W. Sociodemographic predictors of antenatal and postpartum depressive symptoms among women in a medical group practice. J Epidemiol Community Health, v.60, n.3, 221-227, 2006. Disponível em: https://www.ncbi.nlm.nih.gov/pubmed/16476752 Acesso: 14/12/2018.

RODRIGUES, D.P; FERNANDES, A.F.C; SILVA, R.M, RODRIGUES, S.P. O domicílio como espaço educativo para o autocuidado de puérperas: binômio mãe-filho. Texto Contexto Enferm. v.15, n.2, 277-86, 2006.Disponível em: http://www.scielo.br/scielo.php?script=sci_arttext\&pid=S0104-07072006000200012 Acesso: 14/12/2018.

RUSCHI, G.E.C. ET AL. Aspectos epidemiológicos da depressão pós-parto em amostra brasileira. Rev psiquiatra, v.29, n.3, 274-280, 2007. Disponível em: http://www.scielo.br/scielo.php?pid=S0101-

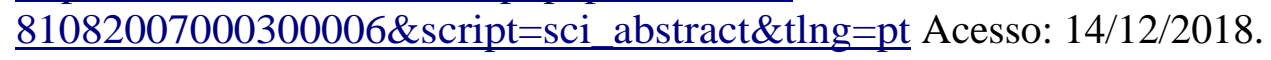


SARAIVA, E.R.A, COUTINHO, M.P.L. A estrutura das representações sociais de mães puérperas acerca da depressão pós-parto. Psico-USF, v.12, n.2, 319-326, 2007. Disponível em: http://pepsic.bvsalud.org/scielo.php?script=sci_arttext\&pid=S141498932008000200003 Acesso: 14/12/2018.

SCHWENGBER, D.D.S; PICCININI, C.A. O impacto da depressão pós-parto para a interação mãe- bebê. Estud psicol (Natal), v.8, n.3, 403-411, 2003. Disponível em: http://www.scielo.br/scielo.php?pid=S1413-

294X2003000300007\&script=sci_abstract\&tlng=pt Acesso: 14/12/2018.

SEGRE, L. S; O'HARA, M. W; ARNDT, S; STUART, S. The prevalence of postpartum depression. The relative significance of three social status indices. Social Psychiatry and Psychiatric Epidemiology, v.42, n.4, 316-321, 2007. Disponível em: https://www.ncbi.nlm.nih.gov/pubmed/17370048 Acesso: 14/12/2018.

SILVA, R; JANSENS, K; SOUZA, L; QUEVEDO, L; BARBOSA, L; MORAES, I; PINHEIRO, R. Sociodemographic risk factors of perinatal depression: A cohort study in the public health care system. Revista Brasileira de Psiquiatria, v.34, n.2, 143-148, 2012. Disponível em: $\quad$ http://www.scielo.br/scielo.php?script=sci_arttext\&pid=S151644462012000200005 Acesso: 14/12/2018.

TACHIBANA, M; SANTOS, L.P; DUARTE, C.A.M. O conflito entre o consciente e o inconsciente na gravidez não planejada . Psychê, v.10, n.19,149-67.2006. Disponível em: http://pepsic.bvsalud.org/scielo.php?script=sci_arttext\&pid=S1415-11382006000300010

Acesso: 14/12/2018.

TANNOUS, L; GIGANTE, L.P; FUCHS, S.C; BUSNELLO, E.D. Postnatal depression in Southern Brazil: prevalence and its demographic and socioeconomic determinants. BMC Psychiatry, $\quad$ v.8, n.1, 2008. 2 Disponível https://bmcpsychiatry.biomedcentral.com/articles/10.1186/1471-244X-8-1.Acesso: 4/12/2018.

TOPIWALA, A; HOTHI, G; EBMEIER, K. P. Identifying patients at risk of perinatal mood disorders. Practitioner, v.256, n.1751, 15-18, 2012. Disponível em: https://www.ncbi.nlm.nih.gov/pubmed/22774377 Acesso: 14/12/2018.

ZANOTTI, D.V; SAITO, K.C; RODRIGUES, M.D; OTANI, M.A.P. Identificação e intervenção no transtorno psiquiátrico e intervenção no transtorno, associadas ao puerpério: a colaboração do enfermeiro psiquiatra. Rev Nursing, v.61, n.6, 36-42, 2003. Disponível em:

http://bases.bireme.br/cgibin/wxislind.exe/iah/online/?IsisScript=iah/iah.xis\&src=google \&base=BDENF\&lang=p\&next Action=lnk\&exprSearch=16505\&indexSearch=ID. Acesso: $14 / 12 / 2018$ 